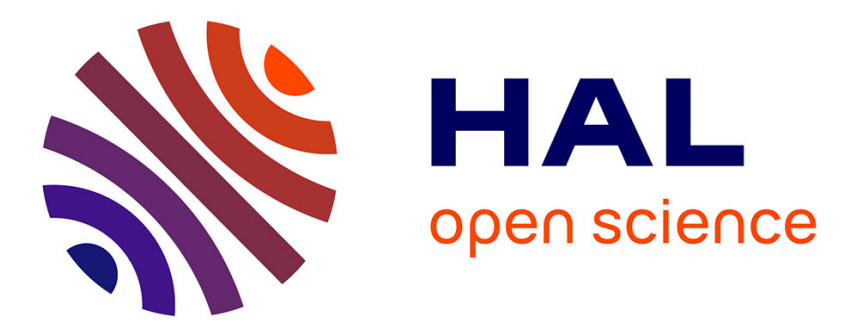

\title{
Optical single-channel color image asymmetric cryptosystem based on hyperchaotic system and random modulus decomposition in Gyrator domains
}

Hang Chen, Li Zhu, Zhengjun Liu, Camel Tanougast, Feifei Liu, Walter

Blondel

\section{To cite this version:}

Hang Chen, Li Zhu, Zhengjun Liu, Camel Tanougast, Feifei Liu, et al.. Optical single-channel color image asymmetric cryptosystem based on hyperchaotic system and random modulus decomposition in Gyrator domains. Optics and Lasers in Engineering, 2020, 124, pp.105809. 10.1016/j.optlaseng.2019.105809 . hal-03487276

\section{HAL Id: hal-03487276 \\ https://hal.science/hal-03487276}

Submitted on 20 Dec 2021

HAL is a multi-disciplinary open access archive for the deposit and dissemination of scientific research documents, whether they are published or not. The documents may come from teaching and research institutions in France or abroad, or from public or private research centers.
L'archive ouverte pluridisciplinaire HAL, est destinée au dépôt et à la diffusion de documents scientifiques de niveau recherche, publiés ou non, émanant des établissements d'enseignement et de recherche français ou étrangers, des laboratoires publics ou privés.

\section{(ㄷ)(1) $\$$}

Distributed under a Creative Commons Attribution - NonCommerciall 4.0 International 


\title{
Optical single-channel color image asymmetric cryptosystem based on hyperchaotic system and random modulus decomposition in Gyrator domains
}

\author{
Hang Chen, ${ }^{1,2^{*}}$ Li Zhu, ${ }^{2}$ Zhengjun Liu, ${ }^{3}$ Camel Tanougast, ${ }^{4}$ Feifei Liu, ${ }^{1}$ and Walter Blondel ${ }^{2}$ \\ ${ }^{I}$ School of Electrical Engineering and Automation, Jiangxi University of Science and Technology, \\ Ganzhou, 341000, China \\ ${ }^{2}$ Université de Lorraine, CNRS, CRAN UMR 7039, Nancy 54000 , France \\ ${ }^{3}$ Department of Automation Measurement and Control, Harbin Institute of Technology, Harbin \\ 150001, China \\ ${ }^{4}$ Université de Lorraine, Laboratoire Conception Optimisation et Modélisation des Systèmes, \\ Metz 57070, France \\ *Corresponding author: hitchenhang@foxmail.com
}

\begin{abstract}
An enhanced optical single-channel asymmetric cryptosystem for color image is proposed by using hyperchaotic system and random modulus decomposition (RMD) in gyrator domains. For increasing the security of the encryption scheme, a novel 4D four-winged hyperchaotic system is utilized to generate several hyperchaotic phases in the encryption approach. Subsequently, the RMD is implemented to obtain the effective trapdoor one-way function in the asymmetric process. Finally, a scrambling encoding algorithm is designed to combine and randomize the intermediate and hyperchaotic data, and the result can be regarded as the ciphertext and private key of the cryptosystem. In the decryption approach, the ciphertext and private key are imported into the input plane of gyrator transform. In fact, the proposed cryptosystem is also applicable in information authentication since the hyperchaotic data is prerequisite both in encryption and decryption approaches. Various numerical simulations are made to demonstrate the validity and capability of the proposed cryptosystem.
\end{abstract}

Keywords: Asymmetric cryptography, color image encryption, optical transform.

\section{Introduction}

With the rapid development of the modern communication techniques, the optical information security technology has drawn wide attention in recent decades due to its parallel processing and high speed processing capabilities. Various optical cryptosystems have been reported since Refrégiér and Javidi first proposed double random phase encoding (DRPE) system in 1995, which can encrypt the image into stationary white noise in $4 f$ optical system [1]. However, DRPE scheme has been verified as vulnerable to different kinds of attack, such as chosen plaintext attack and known plaintext attack, due to its inherent linearity property [2-4]. Subsequently DRPE has been deeply researched and various improved optical cryptosystems have been proposed in other optical transform domains, such as fractional Fourier transform (FrFT), gyrator transform and Fresnel transform to enhance the security and overcome the inherent linearity property [5-18]. Moreover, some other techniques, such as polarized light 
interference [19], randomized lens-phase functions [20] and compressive sensing [21] have also been introduced and utilized to break the linear relationship between the plaintext and ciphertext in optical encryption system. Besides, some random encoding algorithm has also been utilized in the image cryptosystem [22].

Most of the schemes [1-22] described above are symmetric encryption algorithms, in which the decryption key is identical to the encryption key. To overcome the linearity characteristics of DRPE, various asymmetric cryptosystems have been proposed based on phase-truncated Fourier transform (PTFT) [23-25]. However, the PTFT-based asymmetric cryptosystem was proved to be vulnerable to some specific attack using iterative amplitude-phase retrieval algorithm in the further research [26]. Moreover, the iterative phase retrieval algorithm, such as GerchbergSaxton algorithm and Yang-Gu algorithm [27-29], have also been deeply researched and introduced in optical encryption system, which show high robustness against various potential attacks. However, the iterative calculation makes the retrieval algorithm based cryptosystem involved large amount of calculation. Besides the retrieval algorithm, several asymmetric encryption schemes based on equal modulus decomposition (EMD) have been presented in recent years [30-32]. Recently, Wang and his colleagues reported that the attacker is able to obtain precise result from EMD-based encryption system by performing a novel designed attack using phase iterative algorithm [33]. To improve the security of EMD, RMD is investigated, in which a random decomposition idea is utilized in creating the effective trapdoor one-way function. RMD can largely reduce the constraints compared with the EMD and it achieves high robustness while resisting the attack of iterative algorithm [33-34]. However, the RMD is evolved from the original EMD, therefore the parameters of RMD may valuable to exhaustive attack. This attack process may waste much time, but it work in theory. Thus, one can introduce the hyperchaotic system into the cryptosystem, since the high randomness and extreme sensitivity to initial condition of hyperchaotic system can enhance the security of the cryptosystem dramatically. Moreover, some hyperchaotic systems have been employed in some cryptosystem to improve the security since the high sensitive characteristic of the initial value in chaotic system can break the linearity relationship [35-42].

In this paper, a single-channel optical color asymmetric cryptosystem using four-winged hyperchaotic system and random modulus decomposition in gyrator transform domain is proposed. Firstly, a 4D four-winged hyperchaotic system is introduced and utilized to generate the chaotic random phase mask (CRPM), which will be regarded as the public key of asymmetric encryption system. The original color image is encode into two-dimensional data and then combined with the random phase by propagating CRPM placed in the light path. Subsequently the image data is transformed as the light field in output plane of the gyrator transform. At the output plane of gyrator transform, the scrambled data is decomposed into two masks by performing RMD. Finally, a newly designed scrambling pixels position operation according to another chaotic random phase is employed to obtain the ciphertext and private key synchronously. In the encryption approach, the chaotic random phase serves as the public key in this cryptosystem. The parameter in gyrator transform and the initial conditions of the hyperchaotic system can be regarded as the extra keys to guarantee the security. Some corresponding numerical simulations are made to validate the performance of the proposed asymmetric cryptosystem.

The rest of the paper is organized as follow: In Section 2, the proposed cryptosystem is introduced in detail. In Section 3, numerical simulation results are made to demonstrate the validity of the algorithm. Concluding remarks are summarized in the final section. 


\section{Optical color image asymmetric cryptosystem}

Firstly, the 4D four-winged hyperchaotic system, random modulus decomposition and scrambling pixels position operation are introduced briefly, respectively. Then, the gyrator transform and its optical implementation are presented. Finally, the complete asymmetric encryption algorithm is addressed in detail.

\subsection{Hyperchaotic system and scrambling operation}

The highly unpredictable and random-like behavior of the hyperchaotic signals are the most attractive features in cryptography. For the hyperchaotic system, the extreme sensitivity to initial conditions and parameters can improve the security of the data against the brute force attack. It is believes that the performance of higher dimensional chaotic systems (with higher dimensional attractors) are much better in image encryption, therefore a 4D four-winged hyperchaotic system is considered and utilized in this paper and the system can be modeled as follows [43-44]

$$
\left\{\begin{array}{c}
\dot{x}=a x-y z, \\
\dot{y}=b y z-c u+x z, \\
\dot{z}=x y-d z, \\
\dot{u}=y+k u .
\end{array}\right.
$$

where $a, b, c, d$ and $k$ are constant parameters of the hyperchaotic system. The symbol '., represents the operation of derivation. When $a=3, b=-8, c=d=5$ and $k=0.5$, the Lyapunov exponents of the hyperchaotic system are $\lambda_{1}=0.32, \lambda_{2}=1.23, \lambda_{3}=-0.81$ and $\lambda_{4}=-11.04$. Apparently, the first and second Lyapunov are larger than 0 , which means the system is in hyperchaotic state. In calculation, the initial conditions are set as $x_{0}=y_{0}=z_{0}=u_{0}=-10$, and the four-winged $(x-y)$ hyperchaotic Lorenz attractors is illustrated in Fig.1.

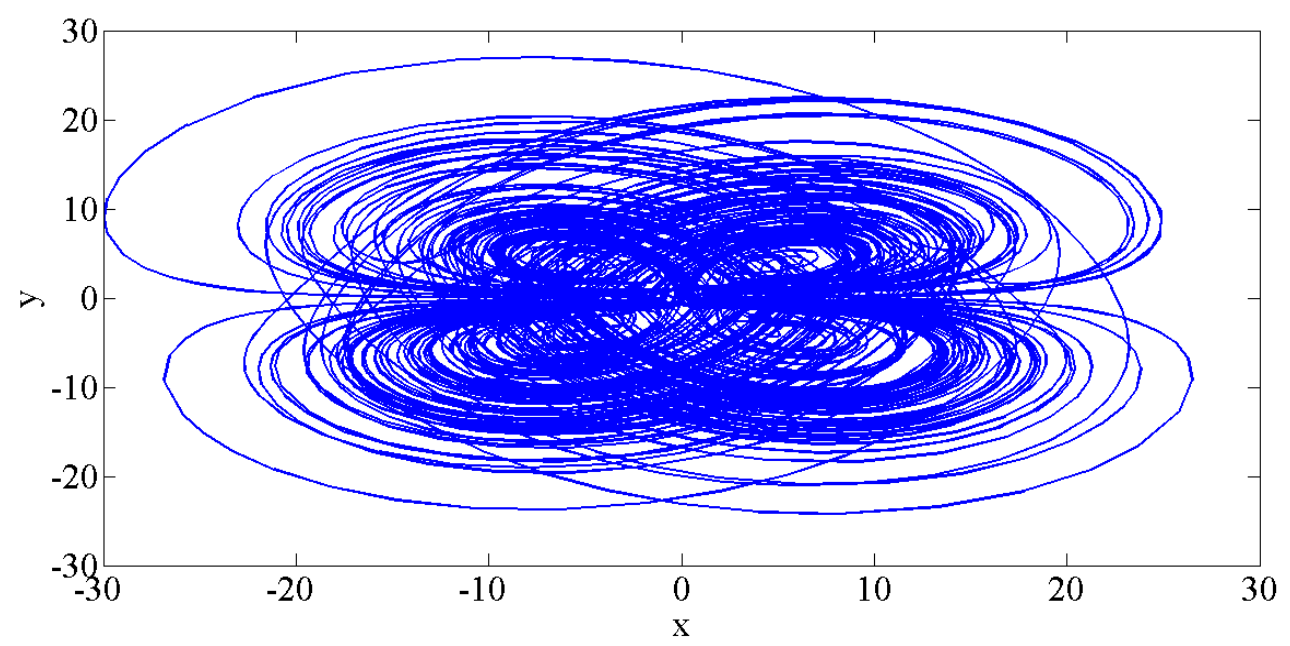

Fig.1 The hyperchaotic Lorenz attractor in x-y plane. 
To obtain the chaotic data for the following encryption process, the hyperchaotic sequences can be generate by the following operation

$$
s_{i}^{*}=s_{i} \times 10^{4}-\operatorname{round}\left(s_{i} \times 10^{4}\right), \quad(i=1,2,3,4) .
$$

where $s_{i}$ denotes the original chaotic sequence from the hyperchaotic system described in Eq.1, which can be also expressed as $x_{i}, y_{i}, z_{i}$ or $u_{i}$. The symbol $s_{i}^{*}$ represents the improved sequences corresponding to $s_{i}$. Therefore, four improved sequences $x_{i}^{*}, y_{i}^{*}, z_{i}^{*}$ and $u_{i}^{*}$ can be obtained by implementing Eq.2. Finally, the obtained improved sequences are converted into two-dimensional format.

To enhance the security of the final ciphertext and private key, the highly unpredictable and random chaotic sequence is considered for designing a scrambling pixels position operation. Here, we suppose the image $I(x, y)$ to be scrambled and the chaotic mask $M(u, v)$ having the same size of $256 \times 256$. Firstly, the chaotic mask $M(u, v)$ is rearranged according to the pixel value, which can be expressed as follows

$$
\left\{\begin{array}{rr}
\left.M^{\prime}\left(u_{t}, v_{k}\right)=\operatorname{sort}\left[\mid M\left(u_{i}, v_{j}\right)\right]\right] & i, j, t, k=1,2,3, \ldots, 256 \\
I^{\prime}\left(x_{t}, y_{k}\right)=\operatorname{array}\left[M^{\prime}\left(u_{t}, y_{k}\right)\right] & t, k=1,2,3, \ldots, 256
\end{array}\right.
$$

where the parameters ' $i$ ', ' $j$ ', ' $t$ ' and ' $k$ ' represent the pixel position before and after the rearrangement. The symbol || indicates the pixel values. In addition, 'sort' and 'array' denote the operation of rearrangement by size and by the specified position. Therefore, the original image $I(x, y)$ can be scrambled by the position of the random chaotic mask.

In the following step, two threshold pixel value $V_{1}$ and $V_{2}$ in the chaotic mask $M^{\prime}\left(u_{t}, v_{k}\right)$ are selected randomly. According to the position corresponding to the threshold $V_{1}$ and $V_{2}$, the scrambled image $I^{\prime}(x, y)$ is divided into three sections. Finally, the pixels in these sections are synchronously arranged in parallel and vertical, as depicted in Fig.2. The mathematical definition of the scrambling pixels position operation can be written as follows

$$
\left\{\begin{array}{lrl}
I_{1}(x, y)=I^{\prime}\left(x_{t}, y_{k}\right), & & \left|M\left(u_{t}, v_{k}\right)\right| \leq V_{1} \\
I_{2}(x, y)=I^{\prime}\left(x_{t}, y_{k}\right), & V_{1}<\left|M\left(u_{t}, v_{k}\right)\right| \leq V_{2} \\
I_{3}(x, y)=I^{\prime}\left(x_{t}, y_{k}\right), & \left|M\left(u_{t}, v_{k}\right)\right|>V_{2}
\end{array}\right.
$$

where $I_{1}(x, y), I_{2}(x, y)$ and $I_{3}(x, y)$ are the three sections of the scrambling image according to the threshold $V_{1}$ and $V_{2}$, respectively. 


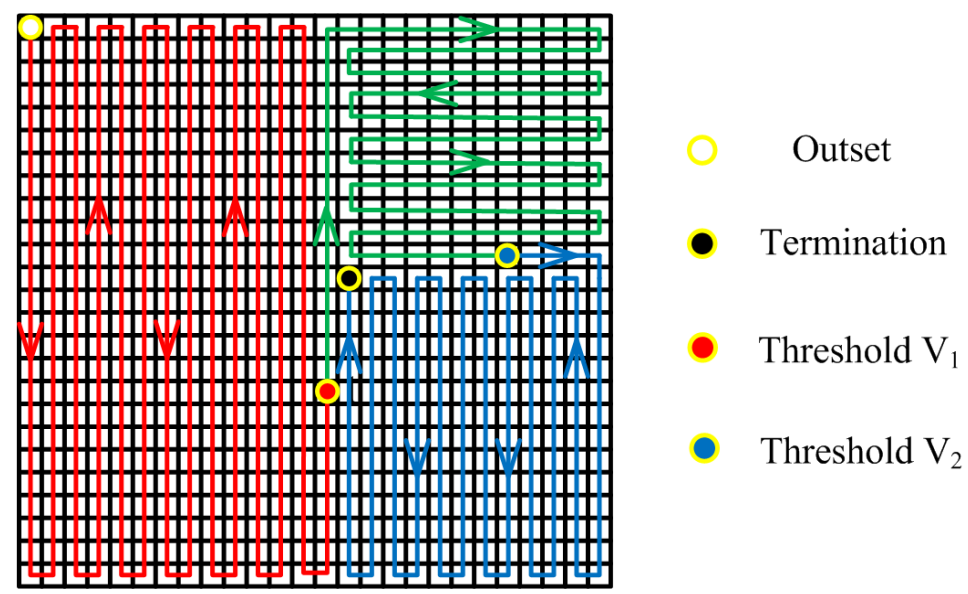

Fig.2 Schematic diagram of the scrambling operation.

\subsection{Random modulus decomposition}

Different from the equal modulus decomposition, random modulus decomposition can highly reduce the constraints since the modulus are not identical according the decomposition theory. The RMD is a kind of unequal modulus decomposition, in which the spectrum is randomly divided into two complex value masks [33]. Therefore, one two-dimensional image can be divided into two statistically independent masks randomly. To simplify the illustration, one complex number in two-dimensional Cartesian coordinate is considered as a vector $I$ and it is divided into two vectors randomly as shown in Fig.3.

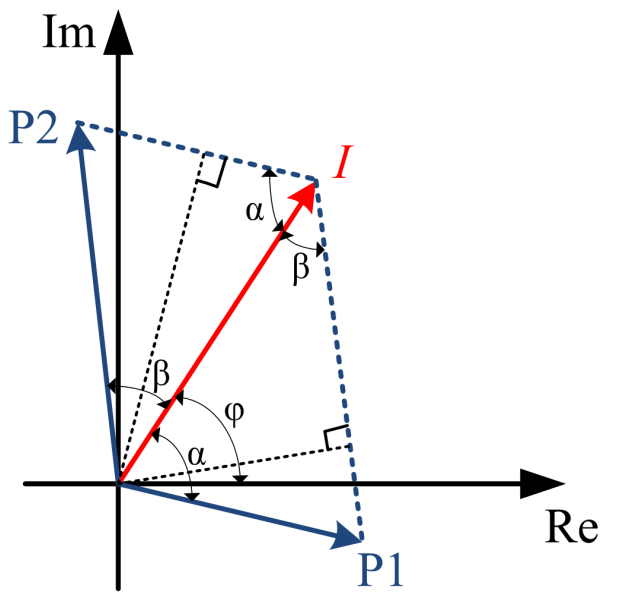

Fig.3 Schematic (vector) representation of the decomposition process in RMD.

Suppose that $I(u, v)$ in Fig.3 is an image, the function $\alpha(u, v), \beta(u, v)$ and $\varphi(u, v)$ can be expressed as equation 


$$
\left\{\begin{array}{c}
\alpha(u, v)=2 \pi \times \operatorname{rand}(u, v) \\
\beta(u, v)=2 \pi \times \operatorname{rand}(u, v) \\
\varphi(u, v)=\arg \{I(u, v)\}
\end{array}\right.
$$

where the function $\operatorname{rand}(u, v)$ denotes a random matrix satisfying normal distribution in the interval $[0,1]$ and the symbol 'arg' represents the argument function. Besides, the amplitude of $I(u, v)$ is given by $A(u, v)=|I(u, v)|$. According to Fig.3, the result of the decomposition masks $P 1(u, v)$ and $P 2(u, v)$ can be obtained by geometrical deduction as follows

$$
P 1(u, v)=\frac{A(u, v)[\sin [\beta(u, v)]}{\sin [\alpha(u, v)+\beta(u, v)]} \exp \{i[\varphi(u, v)-\alpha(u, v)\}
$$

and

$$
P 2(u, v)=\frac{A(u, v)[\sin [\alpha(u, v)]}{\sin [\alpha(u, v)+\beta(u, v)]} \exp \{i[\varphi(u, v)+\beta(u, v)]\}
$$

where the $A(u, v)$ and $\varphi(u, v)$ are the amplitude and phase of $I(u, v)$, respectively. In addition, $\theta(u, v)$ is a random function distributed uniformly in interval $[0,2 \pi]$. Besides, the phase function $\alpha(u, v)$ and $\beta(u, v)$ are given by $\alpha(u, v)=2 \pi \times \operatorname{rand}(u, v)$ and $\beta(u, v)=2 \pi \times \operatorname{rand}(u, v)$ as illustrated in Eq.5, respectively.

\subsection{Optical gyrator transform}

In signal processing filed, the optical gyrator transform can be regarded as a kind of linear canonical integral transform which produces the twisted rotation in position-spatial frequency planes of phase space [45]. In optical implementation, a coherent optical system consisting of six thin cylinder lenses can be considered to realize the optical gyrator propagation [46-47]. In fact, the gyrator transform has only two-dimensional format and the mathematical definition of gyrator transform for the function $I(x, y)$ can be expressed as

$$
\begin{aligned}
F(u, v) & =G^{\alpha}[I(x, y)](u, v) \\
& =\frac{1}{|\sin \alpha|} \iint I(x, y) \exp \left[i 2 \pi \frac{(x y+u v) \cos \alpha-x v-y u}{\sin \alpha}\right] \mathrm{d} x \mathrm{~d} y,
\end{aligned}
$$

where $I(x, y)$ and $F(u, v)$ represent the input and output of gyrator transform, respectively. $x$ and $y$ are the input coordinates at spatial domain, while $u$ and $v$ denote the output coordinates in gyrator domain. Besides, the parameter ' $\alpha$ ' is the rotation angle of gyrator transform, which usually serves as an additional key in encryption system. When $\alpha=\pi / 2$, the gyrator transform reduces Fourier transform with the rotation of coordinates at $\pi / 2$. Finally, the inverse transform of gyrator transform with rotation angle $\alpha$ is transform with rotation angle $-\alpha$ or $2 \pi-\alpha$. Also, 
the gyrator transform has the property of index additive and periodic with the parameter $\alpha$. The optical gyrator transform is utilized to complete the proposed asymmetric cryptosystem.

\subsection{Asymmetric cryptosystem}

The flowchart of the intact proposed cryptosystem is depicted in Fig.4. To implement the proposed cryptosystem, the 4D four-winged hyperchaotic system, optical gyrator transform, RMD and pixels scramble operation are utilized. As shown in Fig.4, the hyperchaotic system described in Section 2.1 is performed to generate the chaotic data. Part of the chaotic data is encoded into the RGB format which having the same size of the original color image. Note that the chaotic data used here serves as the public key of the proposed encryption system. The secret image is combined with the public key and then encoded into two-dimensional format. Subsequently, the intermediate variables are imported into the input plane of gyrator transform and propagate in the gyrator domain.

In the following step, the result of the gyrator transform is analytically decomposed into two masks $P 1$ and $P 2$ by performing RMD. Finally, with the help of chaotic data and two threshold, the pixels position scrambling operation described in Section 2.1 is performed with $P 1$ and $P 2$ synchronously to obtain the ciphertext and private key. The final ciphertext and private key are both color random mask as displayed in Fig.4. What is more, the initial condition of the hyperchaotic system, parameter $\alpha$ in gyrator and two thresholds $V_{1}$ and $V_{2}$ can be regarded as the extra keys of the encryption scheme to enhance the security. Accordingly, the private key and all the additional keys are required to retrieve the secret image. In the next section, various experiments are made to test the validity of the private and extra keys in retrieve approach.

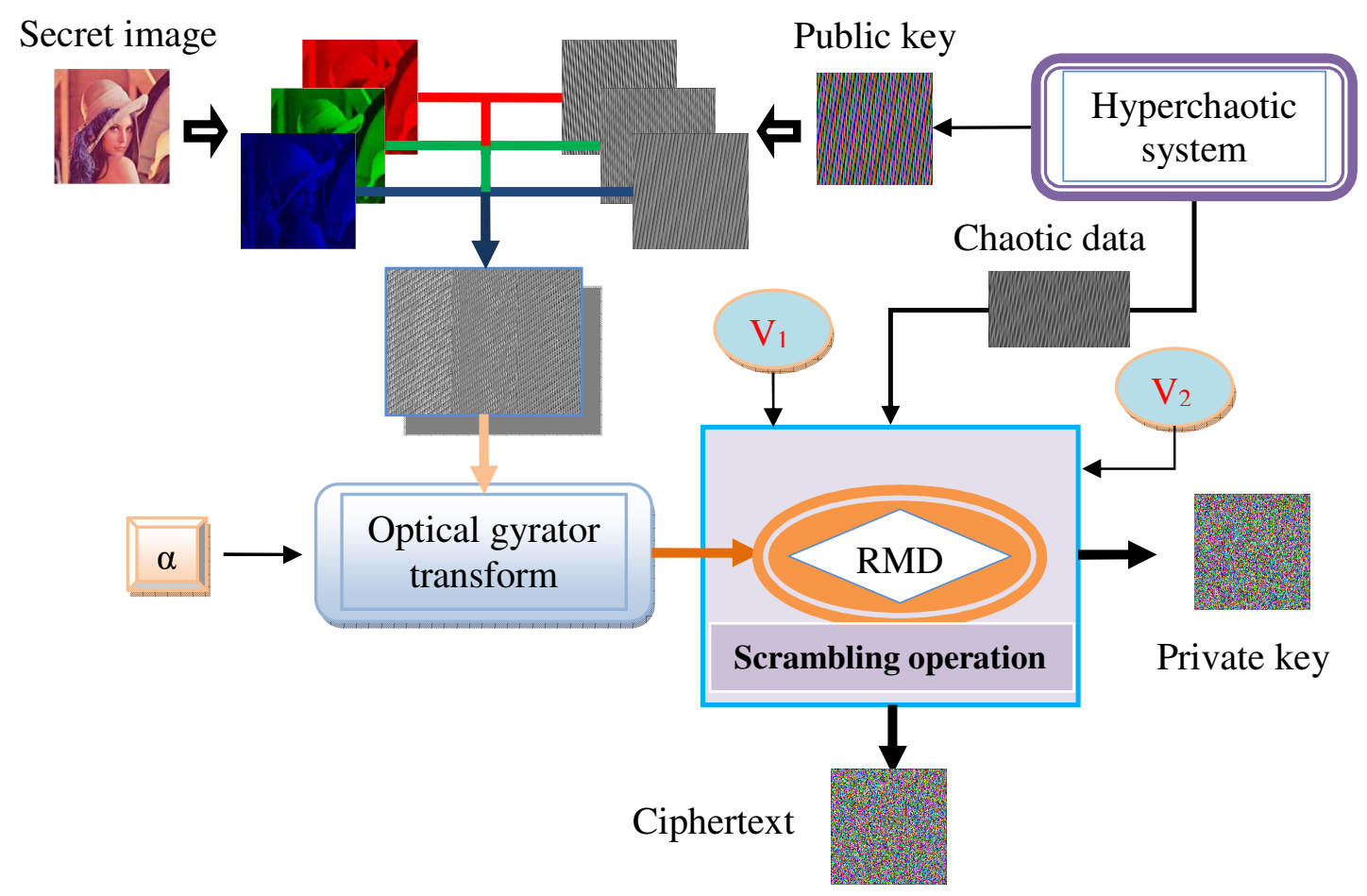


Fig.4 The flowchart of the asymmetric cryptosystem

Since the proposed cryptosystem is asymmetric scheme, the ciphertext cannot be recovered without the private key and public key. At the first step of the decryption process, the ciphertext and private key are calculated by the inverse pixels scrambling operation and RMD with two correct thresholds $V_{1}$ and $V_{2}$, respectively. Subsequently, the retrieval $P 1$ and $P 2$ are transmitted into two spatial light modulator (SLM) and then two beams interfere in a beam splitter (BS). After the beams propagated from the inverse gyrator lenses, the amplitudes and phases are recorded in the output plane and finally decoded back to RGB format image.

The encryption/decryption processes of the intact cryptosystem can be implemented by the electro-optical setups as depicted in Fig.5(a) and Fig.5(b), respectively. The optical gyrator transform and its inverse version are achieved in gyrator lenses system as illustrated in Fig.5(a) [47]. Furthermore, the calculation of hyperchaotic system and scrambling operations is accomplished in the computer. Furthermore, the data communication between the computer and optical system is achieved by SLM and CCD as shown in Fig.5. In the output plane of the gyrator transform, the $\mathrm{CCD}$ and in-line holography technique is introduced to record the amplitude and phase, respectively. Note that, different from the symmetric encryption algorithm, the decryption process of asymmetric scheme cannot fulfill by the inverse version of the encryption process. At the aspect of decryption approach of our proposed cryptosystem, the input plane of the electro-optical setups should be changed to two SLM for modulating the private and ciphertext synchronously and then two beams interfere in the beam splitter (BS) as displayed in Fig.5(b). The beams, then, transformed by inverse gyrator transform and received by CCD at the output plane. It is worth note that the proposed algorithm can serve in the hardware-based cryptographic.

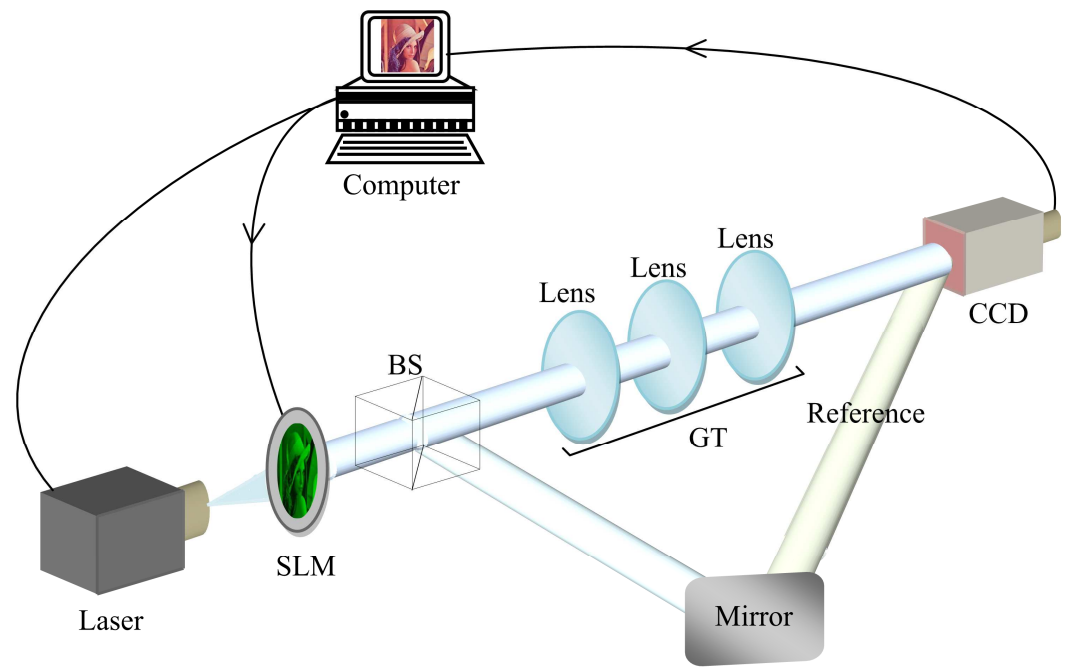

(a) 


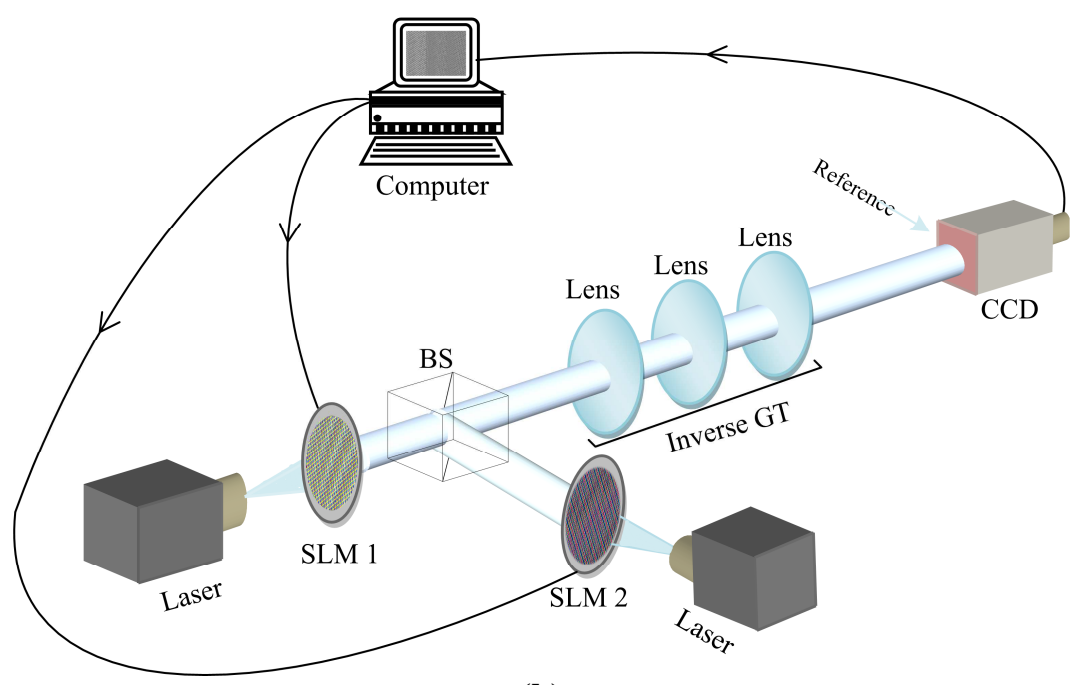

(b)

Fig.5 The electro-optical setup used for the proposed cryptosystem: (a) encryption setup and (b) decryption setup.

\section{Numerical simulation}

Various numerical simulations are made to testify the security and robustness of the proposed cryptosystem in this section. A test color image 'Lena' having $256 \times 256$ pixels is considered as the original secret image to be encrypted, which is displayed in Fig.6(a). In addition, the twodimensional encoded image combined with the original color image and the chaotic data is illustrated in Fig.6(b), which has the size of $384 \times 512$. Accordingly, the image can be propagated and transformed in the single channel optical gyrator domain. At the aspect of chaotic data generated by the hyperchaotic system, the data format is encoded according to the original image. Therefore, after the newly designed scrambling pixels position operation, the function will be converted back to RGB format. Simultaneously, in calculation the initial condition of the hyperchaotic system is set as $x_{0}=y_{0}=z_{0}=u_{0}=-10$ as mentioned before. Besides, the rotation angle in gyrator transform and the two thresholds in scrambling algorithm, which are serve as the extra keys are set as $0.8,-8.1331$ and -10.4342 in simulation. The responding ciphertext by using the condition described above is displayed in Fig.7(a), which is random pattern. Concerning to the decrypted process, the secret image can be retrieval only if the decryption setup and all the keys are correct. By using the proper parameters and keys, the decrypted image is depicted in Fig.7(b), which is identical to the original image in human vision. It is worth note that the decrypted image contains speckle when we used the virtual optical system because of the system noise [19]. The result illustrated in Fig.7(b) is executed with a digital system. Moreover, the quantitative analysis will be presented in the following subsection.

Since the result of gyrator transform is complex function, the simulation results shown in this paper is amplitude part. In calculation, the experiments results are executed by a computer with 2 Gigabytes memory under Windows 7 system. 


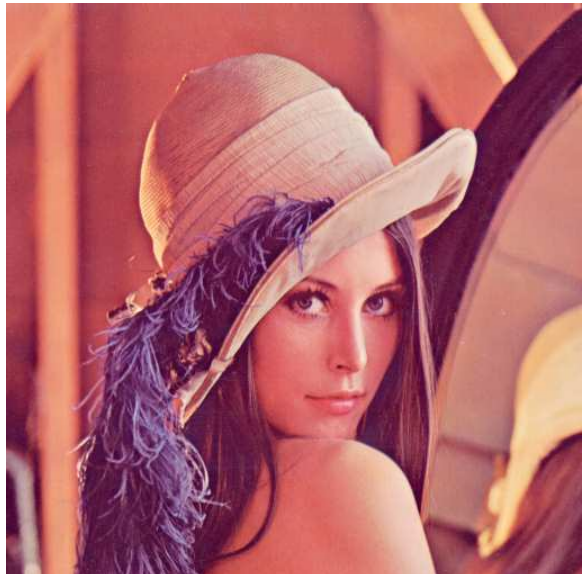

(a)

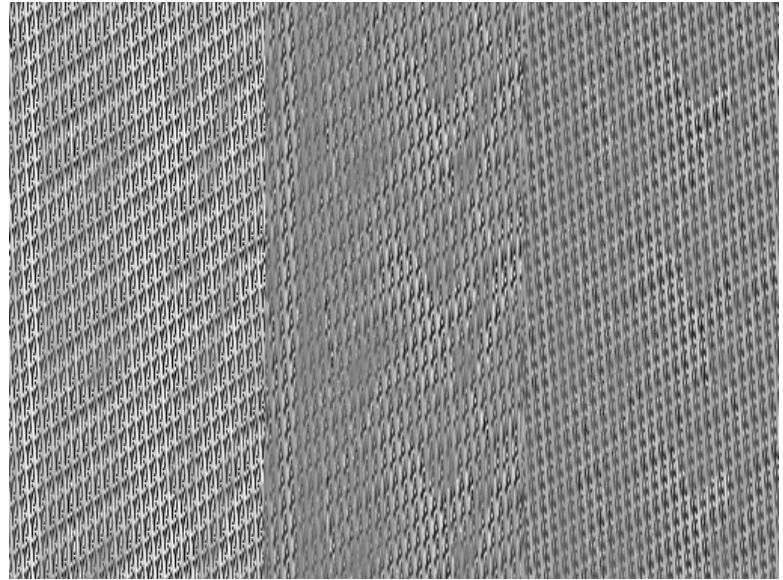

(b)

Fig.6 (a)The original color image test in the experiments and (b) the encoded image combined with the original image and chaotic data.

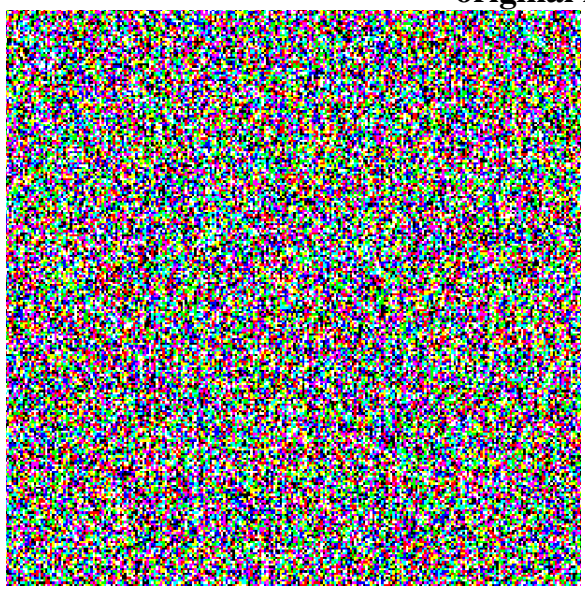

(a)

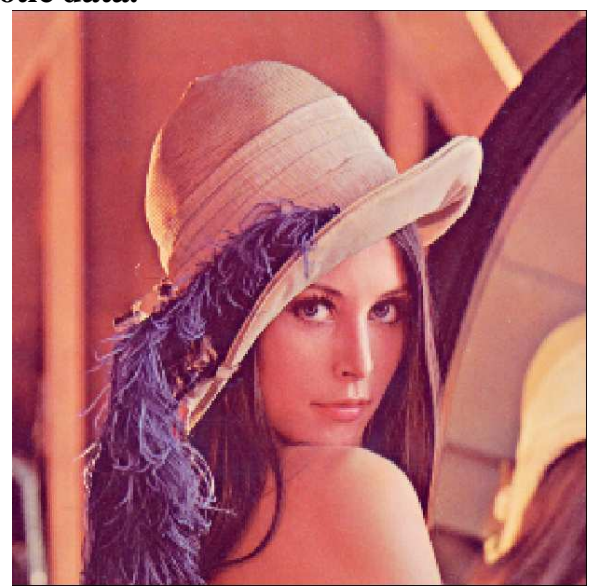

(b)

Fig.7 The encryption/decryption results: (a) the ciphertext and (b) decrypted result.

Apparently, the secret image cannot be recovered by the inverse encryption approach since the RMD leads to the effective trapdoor one-way function, which highly improves the security of the cryptosystem in resisting the attack of iterative algorithm. One can decrypt the original image by employing the setup illustrated in Fig.5(b) with the ciphertext, private key and all the extra keys. Some decryption attempts with the incorrect keys or missing part of the keys are considered to test the proposed scheme in the following subsection. First of all, to distinguish the difference degree between the original and decrypted image, the peak-signal-to-ratio (PSNR) function is introduced. The mathematical definition of PSNR can be written as follows as

$$
\operatorname{PSNR}\left(I_{o}, I_{d}\right)=10 \times \log _{10} \frac{255^{2} M \times N}{\sum_{\forall x, y}\left[I_{d}(x, y)-I_{o}(x, y)\right]^{2}}(\mathrm{~dB}) .
$$

where the input function $I_{o}(x, y)$ and $I_{d}(x, y)$ represent the original image and its decrypted pattern, respectively. Furthermore, the parameters $M$ and $N$ stand for the size in input 
functions. In simulation, the large PSNR value indicates higher similarity between the two input images. Note that the PSNR function will be used to verify the sensitivity of the additional key.

The capability of the private key on protecting the original image is test first. In fact, it is impossible to retrieve the secret image without the private key or using an incorrect key due to its strong randomness. Firstly, two decryption experiments are executed to show the recovered results by using incorrect private key and without private key, respectively. Here, we suppose that the intact encryption/decryption processes are known by the attacker and the cryptosystem is attack using a wrong private key. In calculation, we use a random matrix satisfying uniform distribution in the interval $[0,1]$ instead of the private key. The corresponding retrieval results are displayed in Fig.8(a) and Fig.8(b), respectively. According to the noise-like images depicted in Fig.8, the original color image is completely under protection. Some other random data are also utilized to decrypt the image and obtain the similar results.

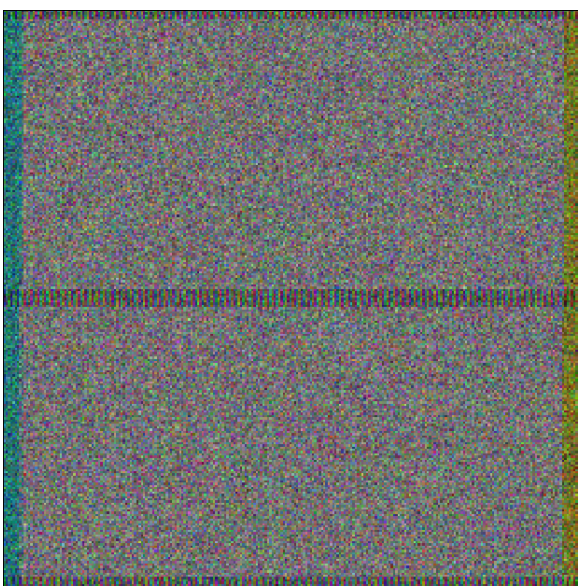

(a)

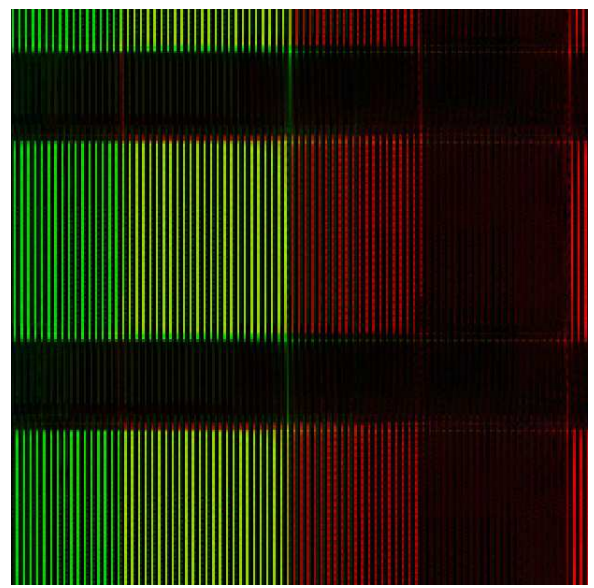

(b)

Fig.8 The decrypt results: (a) using incorrect private key and (b) missing private key.

Concerning to the additional key, the security of the thresholds designed in the pixels scrambling algorithm are also examined. As mentioned above, two pixel position 60000 and 110000 are chosen and the corresponding pixels value (thresholds) are $V_{1}=-8.1331$ and $V_{2}=-10.4342$. Subsequently, the encrypted data is attempted to decrypt by using wrong thresholds and correct keys (including the private key and extra keys). The corresponding decrypted image is illustrated in Fig.9(a), which is blurred image and the secret information is under protected. Moreover, a worse situation is considered. We suppose that the illegal user filch all the private key and extra key, and obtain the two false threshold positions 59999 and 109999 which are very close to the 60000 and 110000 . Under these circumstances, the corresponding recovered image is depicted in Fig.9(b), which is a random-like image even the positions are very close to the real one. Apparently, the noise pattern indicates the high security and sensitivity of the thresholds in protecting the information. 


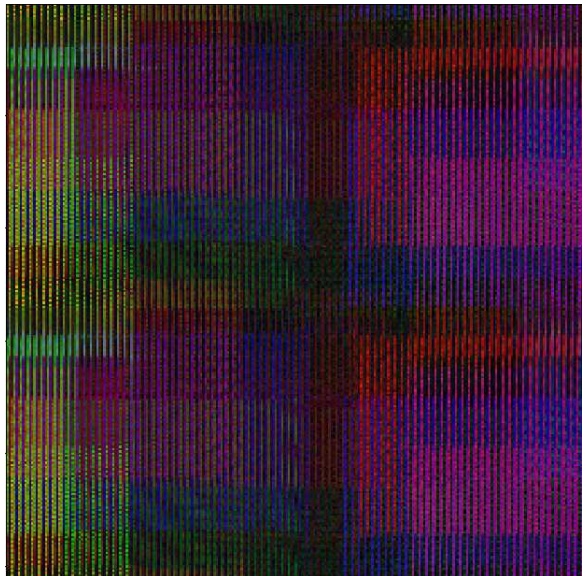

(a)

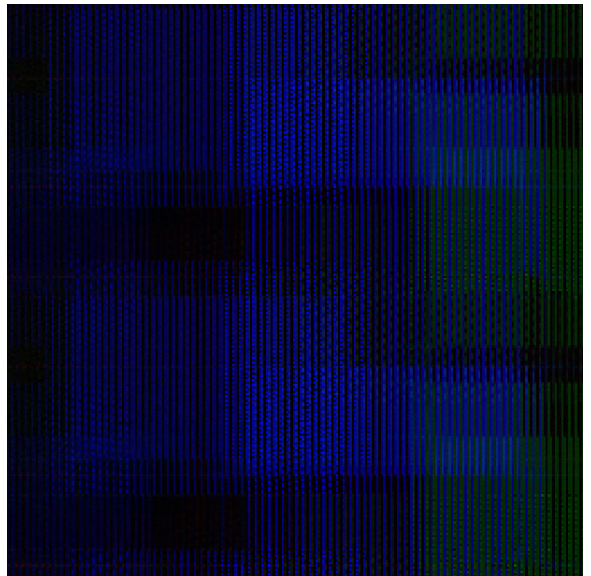

(b)

Fig.9 The decrypt results: (a) using incorrect thresholds and (b) using false threshold positions which are very close to the real one.

Furthermore, as one of the additional key of the cryptosystem, the contribution of the rotation angle $\alpha$ in optical gyrator transform is also test. When all the other keys are known by the attacker, the extra key $\alpha$ is test in the decryption process. In this experiment, the decryption approach is implemented 141 times by using variable $\alpha$ and the corresponding PSNR curve between the secret image and decrypted image is displayed in Fig.10. In calculation, the rotation angle $\alpha$ is changed from 0.5 to 1.2 with the sampling step length 0.005 . Apparently, the parameter $\alpha$ is sensitive enough as an extra key to protect the original image. Two decrypted image by using incorrect keys $\alpha=0.795$ and $\alpha=0.805$ are illustrated in Fig.10, which involved severe noise even the keys are very close to the correct one. Besides, suppose the illegal user try to decrypt the image by using a random key, $\alpha=0.595$ and $\alpha=0.995$ for example, the attacked results are blurred images and the secret information cannot be recognized completely. The original image is perfectly decrypted when the rotation angle $\alpha$ equal to 0.8 .

At the aspect of the contribution of hyperchaotic data, an experiment is design to test the sensitivity of the initial condition. As mentioned above, a minor change of the initial condition in the hyperchaotic system will cause huge and unpredictable change of the attractors. To quantify this unpredictable change caused by the attractors, the Normalized cross correlation (NCC) is considered and utilized. Firstly, the definition of NCC can be written as

$$
\operatorname{NCC}\left(I_{o}, I_{d}\right)=\frac{\sum I_{o}(x, y) \square I_{d}(x, y)}{\sqrt{\sum I_{o}(x, y) \square I_{o}(x, y)} \times \sqrt{\sum I_{d}(x, y) \square I_{d}(x, y)}}
$$

where the input functions $I_{o}$ and $I_{d}$ are the original image and decrypted image. The value of NCC between two images indicates the image similarity and the higher NCC manifests higher similarity at the range of $[0,1]$.

In the following experiment, the performance of the initial conditions of the hyperchaotic system is checked. Firstly, the secret image is encrypted by using specify initial conditions described in Section 2.1. Subsequently, the encrypted data is attacked by the tampered chaotic 
data generated by different initial conditions, which are very close to real values. Finally, the fake initial conditions and the NCC values between the attacked result and the original image are list in the Table.1, respectively. The tiny change of the conditions makes great difference of the decrypted image which implies the information is well protect. The experiment results displayed in Table 1 demonstrates the high sensitive of the parameters in hyperchaotic system. In fact, each parameters of the hyperchaotic system can serve as an independent key because any change of any condition cause huge difference as shown in Table.1. Furthermore, to illustrate the similarity between the decrypted image and the original image, a normalized cross correlation mesh between the correct decrypted image and original image is drawn in Fig.11. In fact, the normalized cross correlation mesh can be used for image matching, that is, searching for an image with the highest NCC area of a known area as a corresponding match, and then aligning the entire image. The value of NCC equal to 1 indicates the high similarity between these two images. To drawn the mesh, one image is traversing to other image, therefore, the size of the normalized cross correlation mesh is $512 \times 512$. The narrow peak of the cross-correlation matrix occurs indicates the high quality of decryption.

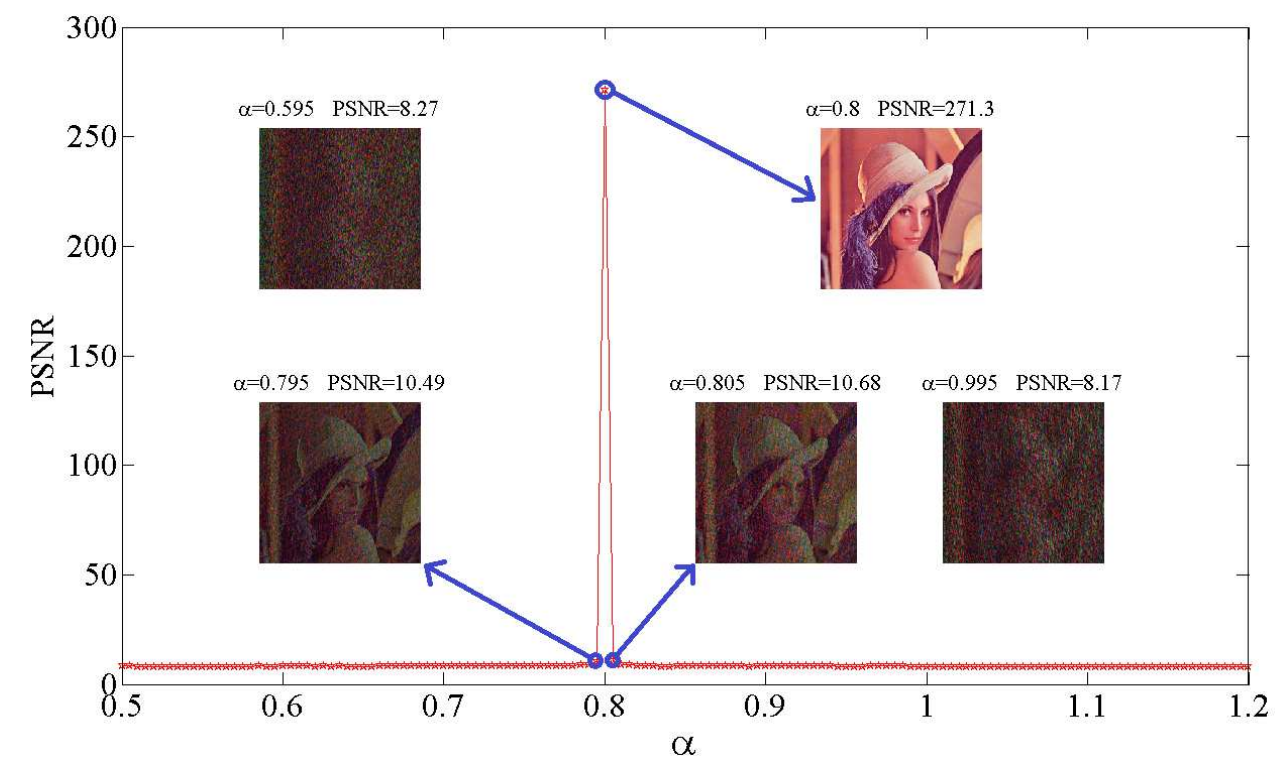

Fig.10 The PSNR curve calculated by variable rotation angle $\alpha$ in gyrator transform.

Table.1 The decryption experiments by using tampered initial conditions.

\begin{tabular}{cccccc}
\hline$x_{0}$ & $y_{0}$ & $z_{0}$ & $u_{0}$ & $P S N R$ & NCC \\
\hline-10 & -10 & -10 & -10 & 271.3 & 1 \\
-9.9999 & -10 & -10 & -10 & 7.6382 & 0.1010 \\
-10 & -9.9999 & -10 & -10 & 9.7318 & 0.2111 \\
-10 & -10 & -9.9999 & -10 & 7.2174 & 0.1003 \\
-10 & -10 & -10 & -9.9999 & 8.0941 & 0.0912 \\
\hline
\end{tabular}




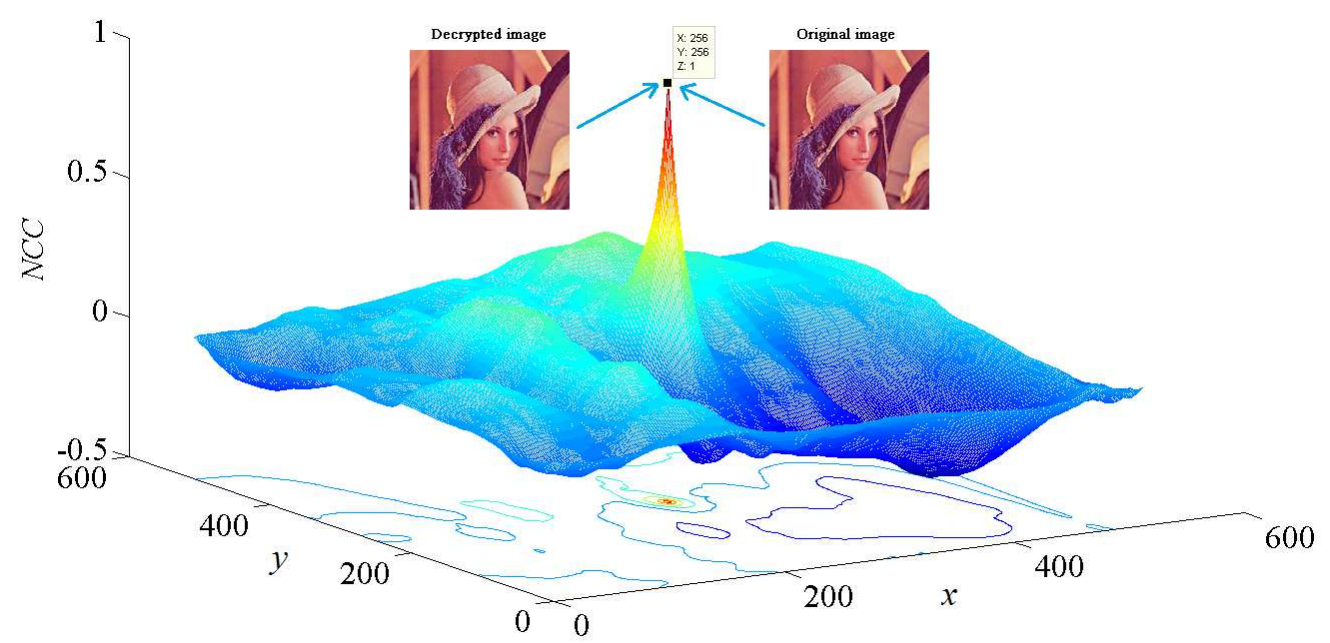

Fig.11 The normalized cross-correlation mesh between the decrypted image and original image.

The occlusion attacks are considered to analyze the robustness of the proposed asymmetric cryptosystem. An original image is encrypted firstly and then the corresponding ciphertext is occluded partly. Subsequently, the occluded ciphertext is decrypted by using all the correct keys (including the private key and additional key) and decryption approach. In calculation, the ciphertext is attacked by occlusion with $1 / 16$ and $1 / 8$, respectively. Note that the occluded part of the ciphertext will be replaced by value 0 in simulation. Under the circumstances mentioned above, the corresponding attack results of the occluded ciphertext are obtained and illustrated in Fig.12(a) and Fig.12(b). According to the decrypted images, the main information and silhouette of the secret image can be recognized in vision, which indicates the well performance of the proposed scheme on resisting the occluded attack. Note that the decrypted image with larger occlusion part has blurrier attack result as depicted in Fig.12.

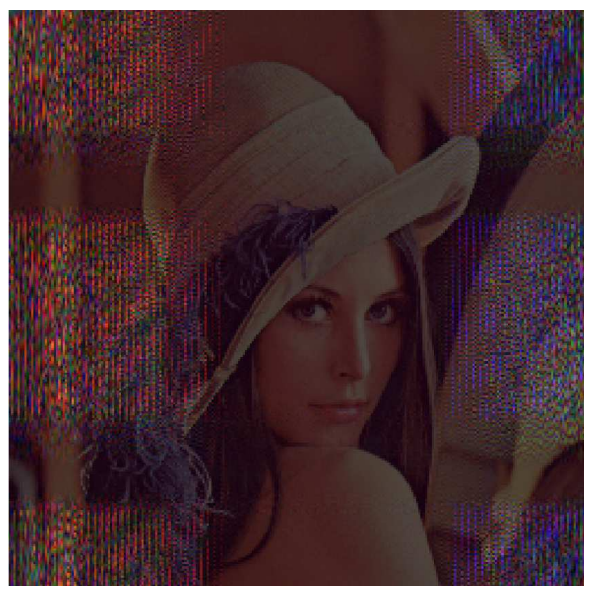

(a)

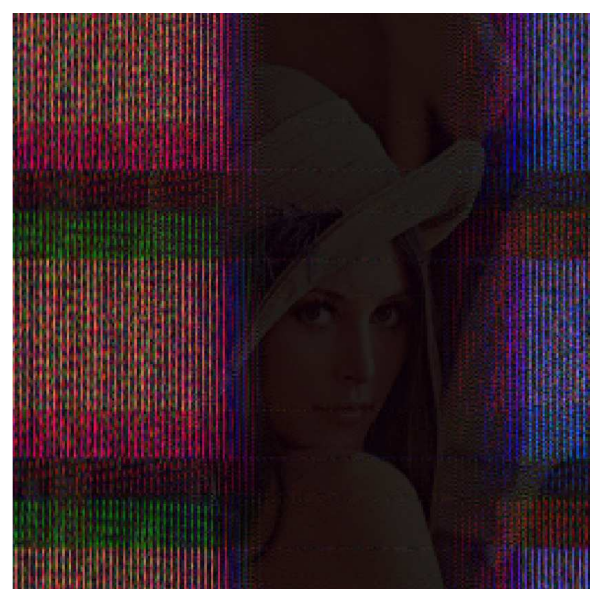

(b)

Fig.12 The test of occlusion attack: (a) attack result with 1/16 occlusion and (b) attack result with 1/8 occlusion. 


\section{Conclusion}

We have presented a single-channel optical color asymmetric cryptosystem by using 4D hyperchaotic system and RMD in gyrator transform domain. The effective trapdoor one-way function generated by RMD extirpates the constraints used for potential attack algorithm. Various highly unpredictable chaotic sequences generated by 4D four-winged hyperchaotic system are utilized in the encoding operation to enhance the security. At the aspect of decryption approach, the high sensitive initial conditions in hyperchaotic system and rotation angle in gyrator transform can be regarded as the additional keys to guarantee the secret information. Various potential attack schemes are considered in the numerical attack experiments and the results have verified the effectiveness, security and robustness of the proposed asymmetric cryptosystem.

\section{Acknowledgment}

This work was supported by the National Natural Science Foundation of China (Grant 61575053), the Program for New Century Excellent Talents in University (NCET-12-0148), and SpectroLive Project form CNRS foundation. The authors wish to thanks Mr. Pierre VARIS for the valuable discussion in pixels position encoding algorithm. The authors are indebted for the anonymous reviewer for the professional and helpful comments and suggestions.

\section{References}

1. Refregier P, Javidi B. Optical image encryption based on input plane and Fourier plane random encoding. Opt Lett 1995; 20: 767-9.

2. Peng X, Zhang P, Wei H, Yu B. Known-plaintext attack on optical encryption based on double random phase keys. Opt Lett 2006; 31: 1044-6.

3. Peng X, Wei H, Zhang P. Chosen-plaintext attack on lensless double-random phase encoding in the Fresnel domain. Opt Lett 2006; 31: 3261-3.

4. Kumar P, Kumar A, Joseph J, Singh K. Vulnerability of the security enhanced double random phase-amplitude encryption scheme to point spread function attack. Opt Lasers Eng, 2012, 50: 1196-201.

5. Unnikrishnan G, Joseph J, Singh K. Optical encryption by double-random phase encoding in the fractional Fourier domain. Opt Lett 2000; 25: 887-9.

6. Situ GH, Zhang JJ. Double random-phase encoding in the Fresnel domain. Opt Lett 2004; 29: 1584-6.

7. Liu Z, Liu S. Random fractional Fourier transform. Opt Lett 2007; 32: 2088-90.

8. Azoug S E, Bouguezel S. A non-linear preprocessing for opto-digital image encryption using multiple-parameter discrete fractional Fourier transform. Opt Commun, 2016; 359: 85-94.

9. Chen W, Chen X. Optical color image encryption based on an asymmetric cryptosystem in the Fresnel domain. Opt Commun 2011; 284: 3913-7.

10. Chen W, Chen X, Sheppard CJR. Optical color-image encryption and synthesis using coherent diffractive imaging in the Fresnel domain. Opt Express 2012; 20: 3853-65.

11. Liu Z, Guo C, Tan J, et al. Securing color image by using phase-only encoding in Fresnel domains. Opt Lasers Eng, 2015; 68: 87-92. 
12. Liu Z, Chen H, Liu T, Li P, Dai J, Sun X, Liu S. Double-image encryption based on the affine transform and the gyrator transform. J Opt 2010; 12: 035407.

13. Abuturab MR. Color image security system based on discrete Hartley transform in gyrator transform domain. Opt Lasers Eng 2013; 51: 317-24.

14. Abuturab MR. An asymmetric single-channel color image encryption based on Hartley transform and gyrator transform. Opt Lasers Eng, 2015; 69: 49-57.

15. Chen H, Tanougast C, Liu Z, Hao B. Securing color image by using hyperchaotic system in gyrator transform domains. Opt Quant Electron, 2016; 48: 396.

16. Meng XF, Cai LZ, Xu XF, Yang XL, Shen XX, Dong GY, Wang YR. Two-step phase shifting interferometry and its application in image encryption. Opt Lett 2006; 31: 1414-6.

17. Zhou N, Hua T, Gong L. Quantum image encryption based on generalized Arnold transform and double random-phase encoding. Quantum Inf Process, 2015; 14: 1193-213.

18. Chen H, Liu Z, Zhu L, Tanougast C, Blondel W. Asymmetric color cryptosystem using chaotic Ushiki map and equal modulus decomposition in fractional Fourier transform domains. Opt Lasers Eng, 2019, 112: 7-15.

19. Zhu N, Wang Y, Liu J, Xie J, Zhang H. Optical image encryption based on interference of polarized light. Opt Express 2009; 17: 13418-24.

20. Kumar P, Kumar A, Joseph J, Singh K. Impulse attack free double-random-phase encryption scheme with randomized lens-phase functions. Opt lett, 2009, 34: 331-3.

21. Deepan B, Quan C, Wang Y, Tay CJ. Multiple-image encryption by space multiplexing based on compressive sensing and the double-random phase-encoding technique. Appl opt, 2014, 53: 4539-47.

22. Chen J, Zhu Z, Zhang L, Zhang Y, Yang B. Exploiting self-adaptive permutation-diffusion and DNA random encoding for secure and efficient image encryption. Signal Processing, 2018, 142: 340-353.

23. Qin W, Peng X. Asymmetric cryptosystem based on phase-truncated Fourier transforms. Opt Lett 2010, 35: 118-20.

24. Wang X, Zhao D. Security enhancement of a phase-truncation based image encryption algorithm. Appl Opt 2011, 50: 6645-51.

25. Rajput S, Nishchal N. Image encryption based on interference that uses fractional Fourier domain asymmetric keys. Appl Opt 2012, 51: 1146-52

26. Wang X, Chen Y, Dai C, Zhao D. Discussion and a new attack of the optical asymmetric cryptosystem based on phase-truncated Fourier transform. Appl opt, 2014, 53: 208-213.

27. Rajput S K, Nishchal N K. Fresnel domain nonlinear optical image encryption scheme based on Gerchberg-Saxton phase-retrieval algorithm. Appl opt, 2014, 53: 418-25.

28. Chen H, Du X, Liu Z, Yang C. Optical color image hiding scheme by using GerchbergSaxton algorithm in fractional Fourier domain. Opt Lasers Eng, 2015, 66: 144-51.

29. Liu W, Liu Z, Wu J, Liu S. Asymmetric cryptosystem by using modular arithmetic operation based on double random phase encoding. Opt Commun, 2013, 301: 56-60.

30. Fatima A, Mehra I, Nishchal N K. Optical image encryption using equal modulus decomposition and multiple diffractive imaging. Journal Opt, 2016, 18: 085701.

31. Chen H, Tanougast C, Liu Z, Sieler Loic. Asymmetric optical cryptosystem for color image based on equal modulus decomposition in gyrator transform domains Opt Lasers Eng, 2017, 93: 1-8.

32. Kumar A, Fatima A, Nishchal N K. An optical Hash function construction based on equal modulus decomposition for authentication verification. Opt Commun, 2018, 428: 7-14. 
33. Wang Y, Quan C, Tay C J. New method of attack and security enhancement on an asymmetric cryptosystem based on equal modulus decomposition. Appl opt, 2016, 55: 67986.

34. $\mathrm{Xu} \mathrm{H}, \mathrm{Xu} \mathrm{W}$, Wang $\mathrm{S}, \mathrm{Wu} \mathrm{S}$. Phase-only asymmetric optical cryptosystem based on random modulus decomposition. Journal Mod Opt, 2018, 65: 1245-52.

35. Chen H, Tanougast C, Liu Z, Hao B. Securing color image by using hyperchaotic system in gyrator transform domains. Opt Quan Elect, 2016, 48: 396.

36. Zhou N, Pan S, Cheng S, Zhou Z. Image compression-encryption scheme based on hyperchaotic system and 2D compressive sensing. Opt Laser Tech, 2016, 82: 121-33.

37. Niyat A Y, Moattar M H, Torshiz M N. Color image encryption based on hybrid hyperchaotic system and cellular automata. Opt Lasers Eng, 2017, 90: 225-37.

38. Cao C, Sun K, Liu W. A novel bit-level image encryption algorithm based on 2D-LICM hyperchaotic map. Signal Process, 2018, 143: 122-33.

39. Zhou N, Chen W, Yan X, et al. Bit-level quantum color image encryption scheme with quantum cross-exchange operation and hyper-chaotic system. Quantum Inf Process, 2018, 17: $1-24$.

40. Xu Q, Sun K, Cao C, Zhu C. A fast image encryption algorithm based on compressive sensing and hyperchaotic map. Opt Lasers Eng, 2019, 121: 203-14.

41. Gopalakrishnan T, Ramakrishnan S. Image Encryption Using Hyper-chaotic Map for Permutation and Diffusion by Multiple Hyper-chaotic Maps. Wireless Pers Commun, 2019: $1-18$.

42. Rakheja P, Vig R, Singh P. An asymmetric hybrid cryptosystem using hyperchaotic system and random decomposition in hybrid multi resolution wavelet domain. Multimed Tools Appl, 2019: 1-26.

43. Zhu C. A novel image encryption scheme based on improved hyperchaotic sequences. Opt Commun, 2012, 285: 29-37.

44. Peng Z, Wang C, Lin Y, Luo X. A novel four-dimensional multi-wing hyper-chaotic attractor and its application in image encryption. Acta Phys Sin, 2014, 63: 240506.

45. Vilardy J M, Millán M S, Pérez-Cabré E. Nonlinear image encryption using a fully phase nonzero-order joint transform correlator in the Gyrator domain. Opt Lasers Eng, 2017, 89: 88-94.

46. Rodrigo J A, Alieva T, Calvo M L. Gyrator transform: properties and applications. Opt express, 2007, 15(5): 2190-203.

47. Rodrigo J A, Alieva T, Calvo M L. Experimental implementation of the gyrator transform. JOSA A, 2007, 24(10): 3135-9. 